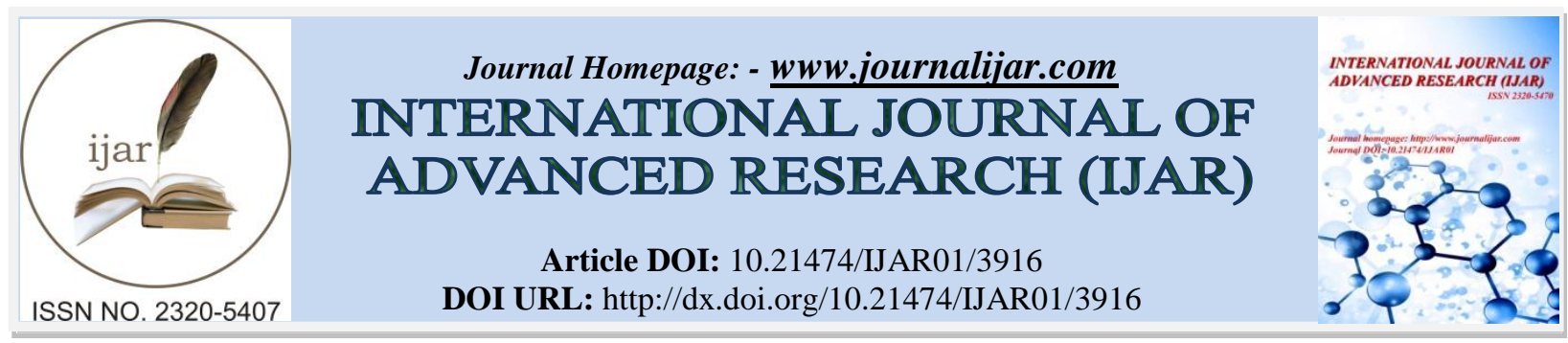

RESEARCH ARTICLE

\title{
MORPHOLOGICAL CHARACTERIZATION OF WEAR DEBRIS FROM THE TRANSMISSION SYSTEM OF DUMPER.
}

\begin{abstract}
"Abhimanyu Sharma, Ashwani Kumar, Sandeep Sharma, Shanti priya vatsh and Dr. Subrata Kumar Ghosh. Department of Mechanical Engineering, Indian Institute of Technology (ISM), Dhanbad.
\end{abstract}

\section{Manuscript Info}

Manuscript History

Received: 19 February 2017

Final Accepted: 16 March 2017

Published: April 2017

Key words:-

Ferrography, morphology, size

parameters, shape parameters.

\section{Abstract}

The aim of this study is to present a morphological analysis of wear debris contained in the used transmission oil of a dumper. The transmission system of the dumper was condition-monitored over a period of 1000 hours through wear debris analysis of used oil. Analytical ferrography has been employed to extract the relevant information like size parameters viz. area, perimeter, ferret's length and shape parameters viz. elongation, aspect ratio and roundness. The wear particles present in the transmission oil have been characterized based on process of generation and shape parameters of wear particles. The experimental results indicate decrease in size parameters with time. However, in case of shape parameters roundness decreases while aspect ratio and elongation show an increase with passage of time. A shift in wear mechanism from normal rubbing wear to sliding wear has also been noticed.

Copy Right, IJAR, 2017,. All rights reserved.

\section{Introduction:-}

Wear particles are produced in all machines from the interaction of contacting surfaces. Any change in the microscopic wear particles, transported by a lubricant from wear sites carry with them important information regarding the condition of machinery[1]. Particles can be classified in terms of their compositional and morphological attributes. The compositional attributes represent whether the particles obtained are metallic or nonmetallic, and if metallic, then ferrous or non-ferrous etc. Particle size, shape, edge detail, color, thickness ratio, and surface texture are the six morphological attributes which have been identified [2].The morphology of wear particles, in terms of their shape and size reflects the complex nature of wear processes involved in particle formation [3, 4].Analytical ferrography can be used to isolate ferrous debris from lubricant and deposit them on a glass substrate for further analysis using a gradient magnetic field. The advantages to this technique are that debris information including color, shape, texture, composition, and size distribution can be obtained [5].

Size parameters describe a geometrical object independently of its shape [6].Various parameters have been used to assess the size of the particles. Some of them discussed in the literature are: Perimeter, Equivalent diameter, ferret's diameter, Martin's diameter, Area, Length (major axis), Width (minor axis) etc. [7].Various size parameters used in this paper are -(a) Area - Area of the two-dimensional projection of the obtained image (b) Major ferret - Ferret diameter along major axis of a particle using the 2D projection (c) Minor ferret - Ferret diameter along minor axis of a particle using the 2D projection (d)Perimeter - Length of a particle's outline using the2D projection. [8].

Corresponding Author:- Abhimanyu Sharma.

Address:- Department of Mechanical Engineering, Indian Institute of Technology (ISM), Dhanbad. 
Shape is described numerically by shape properties. Generally, shape factors describe the tendency of a particle to deviate from an ideal geometrical prototype - often a sphere. Spherical particles are the least abrasive among shapes of comparable size.The shape parameterscharacterize mainly the shape without considering the size.Some of the shape parameters used in this paper are (a) Aspect ratio - It is defined as the ratio of major ferret to minor ferret. (b)Elongation- It is given by the mathematical formula as - $\log _{2}$ [Aspect ratio]. (c) Roundness is given by the mathematical formula $=(4 \times$ Area $) /\left(\pi \times\right.$ Major ferret $\left.{ }^{2}\right)$. The value of roundness varies from $0-1$. The value 1

indicates perfect round shape while 0 indicates highly distorted shape[6].

The authors in this paper have applied size parameters and shape parameters for the characterization of wear particles. In addition to this, variation in the size and shape parameters with time is monitored.

\section{Experimental Procedure:-}

Transmission oil samples were collected from three different dumpers at regular intervals such that there is a time interval of 200 hours between each sample. The oil samples were collected using apump. It was ensured that the suction pipe of the pump was at the center of the sump such suitable quantity of wear particles is included in the sample. If the inlet of the suctionpipe was at the top of the sump very few wear particles would be included in the sample. On the other hand, if the suction pipe is kept at bottom of sump, the number of war particles included in the samples would be very high.

Analytical ferrography was applied for the deposition of wear particles over the glass slide [Ferrogram]. Propane was used as diluting agent in ferrography. The glass slides were then observed under an optical microscope with a magnification of 50X. A charge coupled device camera fitted over the optical microscope was used to capture images of wear particles of various samples.

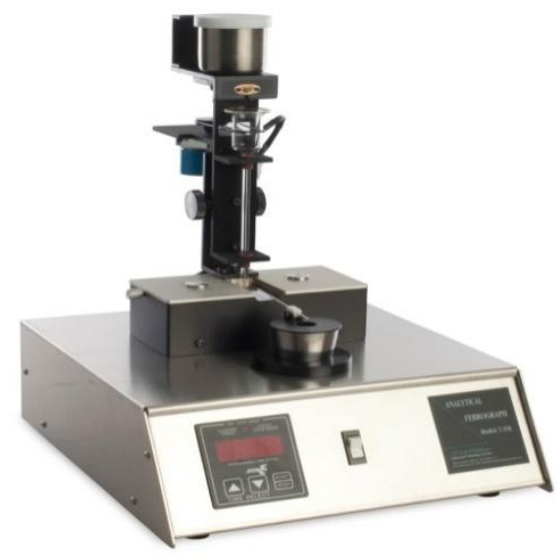

Figure 1:- Analytical ferrograph

These images were then fed into image processing software to obtain the number of wear particles, their size parameters and shape parameters. The size and shape parameters were further analyzed. Tests and analysis were performed on all the three samples but the data and results of only one sample are presented in this paper.

\section{Results and discussions:-}

The transmission oil was monitored over a period of 1000 hours. Oil samples were regularly collected at interval of 200 hours. Variations in number of wear particles, size parameters and shape parameters with time are plotted in the graphs. Out of many size parameters, only area, perimeter, major ferret and minor ferret were selected because they were able to completely specify the size of the wear particle. Similarly roundness and aspect ratio are adequate for describing the shape of the wear particles. Hence other shape parameters were not included in the analysis. 
Table 1:-

\begin{tabular}{|c|c|c|c|c|c|c|c|c|c|c|}
\hline & \multicolumn{10}{|c|}{ Sample-01 } \\
\hline & \multicolumn{2}{|c|}{200 Hrs. } & \multicolumn{2}{|c|}{400 Hrs. } & \multicolumn{2}{|c|}{600 Hrs. } & \multicolumn{2}{|c|}{800 Hrs. } & \multicolumn{2}{|c|}{1000 Hrs. } \\
\hline & Mean & S.D & Mean & S.D & Mean & S.D & Mean & S.D & Mean & S.D \\
\hline Area $\left(\mu \mathrm{m}^{2}\right)$ & 1.92 & 9.6 & 1.6 & 3.4 & 1.4 & 5.7 & 0.73 & 8.3 & 0.4 & 1.5 \\
\hline $\operatorname{Perimeter}(\mu \mathrm{m})$ & 4.35 & 10.1 & 3.71 & 3.8 & 3.48 & 5.8 & 2.51 & 10.2 & 1.86 & 2.4 \\
\hline $\begin{array}{l}\text { Major Ferret } \\
(\mu \mathrm{m})\end{array}$ & 1.45 & 2.5 & 1.25 & 1.2 & 1.216 & 1.6 & 0.97 & 2.2 & 0.74 & 0.8 \\
\hline $\begin{array}{l}\text { Minor Ferret } \\
(\mu \mathrm{m})\end{array}$ & 0.81 & 1.2 & 0.75 & 0.6 & 0.66 & 0.9 & 0.58 & 1.6 & 0.47 & 0.4 \\
\hline Roundness & 0.5547 & 0.1669 & 0.5497 & 0.1804 & 0.5482 & 0.1819 & 0.5416 & 0.1929 & 0.5010 & 0.1622 \\
\hline Aspect ratio & 1.6734 & 0.6664 & 1.6905 & 0.6105 & 1.7132 & 0.6016 & 1.7228 & 0.7198 & 1.8195 & 0.6016 \\
\hline Elongation & 0.7428 & 0.4112 & 0.7575 & 0.4281 & 0.7767 & 0.4273 & 0.7848 & 0.4623 & 0.8635 & 0.4218 \\
\hline
\end{tabular}

Table 1 depicts mean and standard deviation of the size parameters and shape parameter. Variation in the mean of the size and the shape parameters with time can be obtained from the table. The standard deviation signifies how concentrated the data is around the mean.

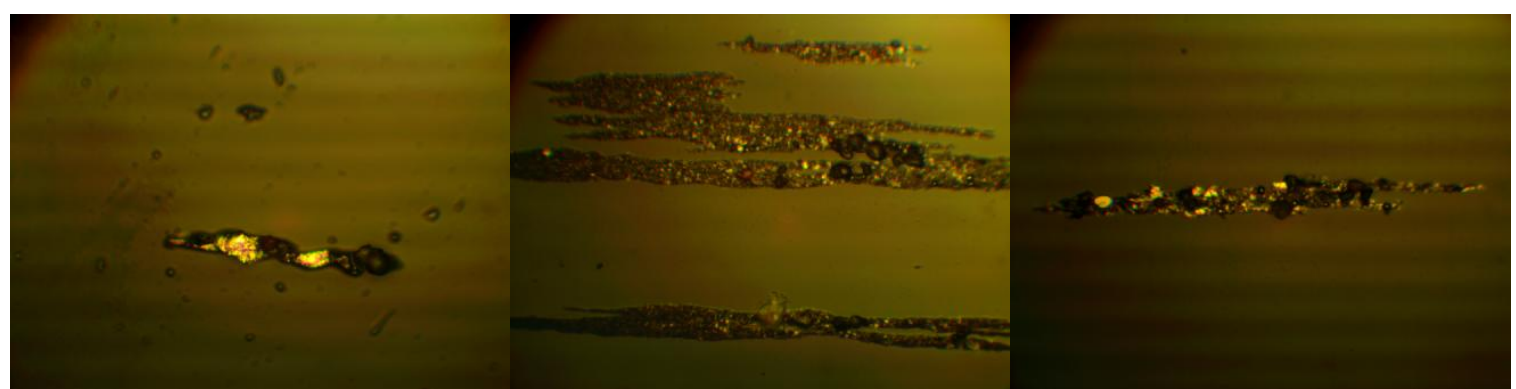

(a)

(b)

(c)

Figure 2:- Type of wear particles

Figure 2(a) denotes sliding wear particle.Sliding wear particles usually have surface striations as a result of sliding movement and an irregular periphery. Figure 2(b) denotes laminar wear particle.Laminar particles usually have a smooth boundary with a relatively random outline and fatigue chunks have one smooth or worked edge with the other edges being jagged.They are very thin in comparison to their major length dimensions with major length to thickness ratio called height aspect ratio (HAR) of approximately 30:1. Figure 2(c) denotes normal rubbing wear particle.Rubbing debris is produced by normal benign wear of a sliding surface.

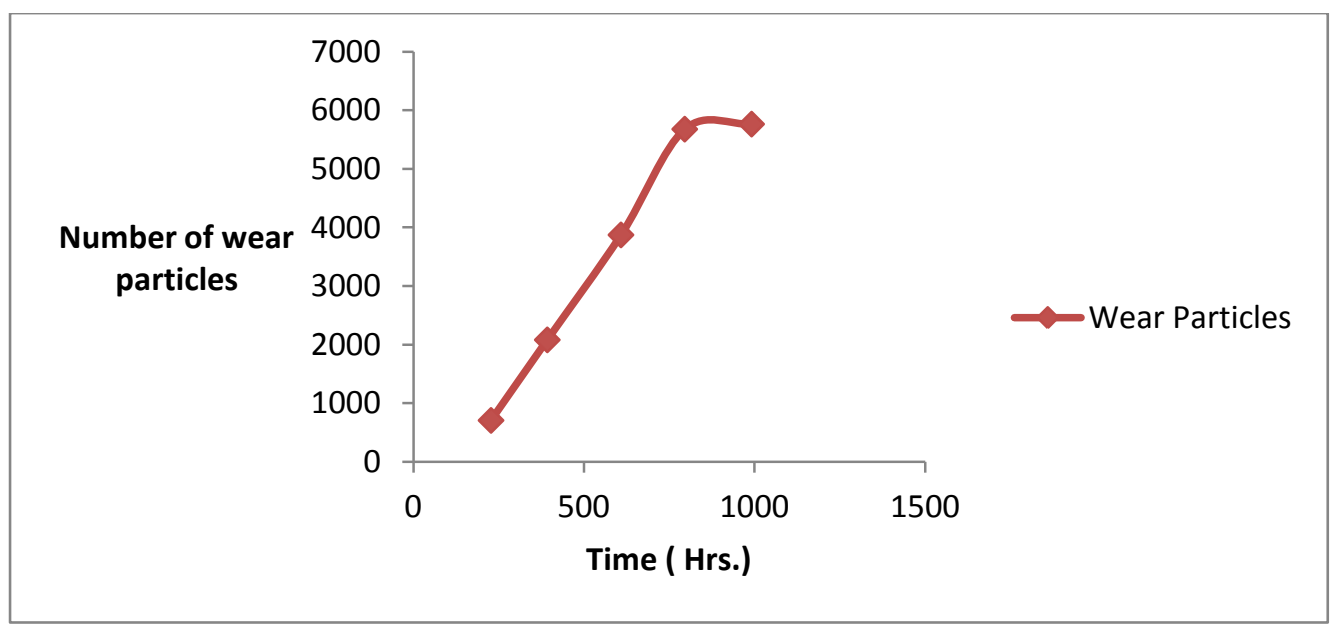

Figure 3:- Variation of number of wear particles with time

Figure 3 denotes variation of number of wear particles with time of usage of transmission oil. It is evident from the figure that the number of wear particles initially increase with passage of time and after a certain time the number of 
wear particles were almost constant with passage of time. In the case of sample 1, the time at which the number of wear particles became constant was 800 hours.

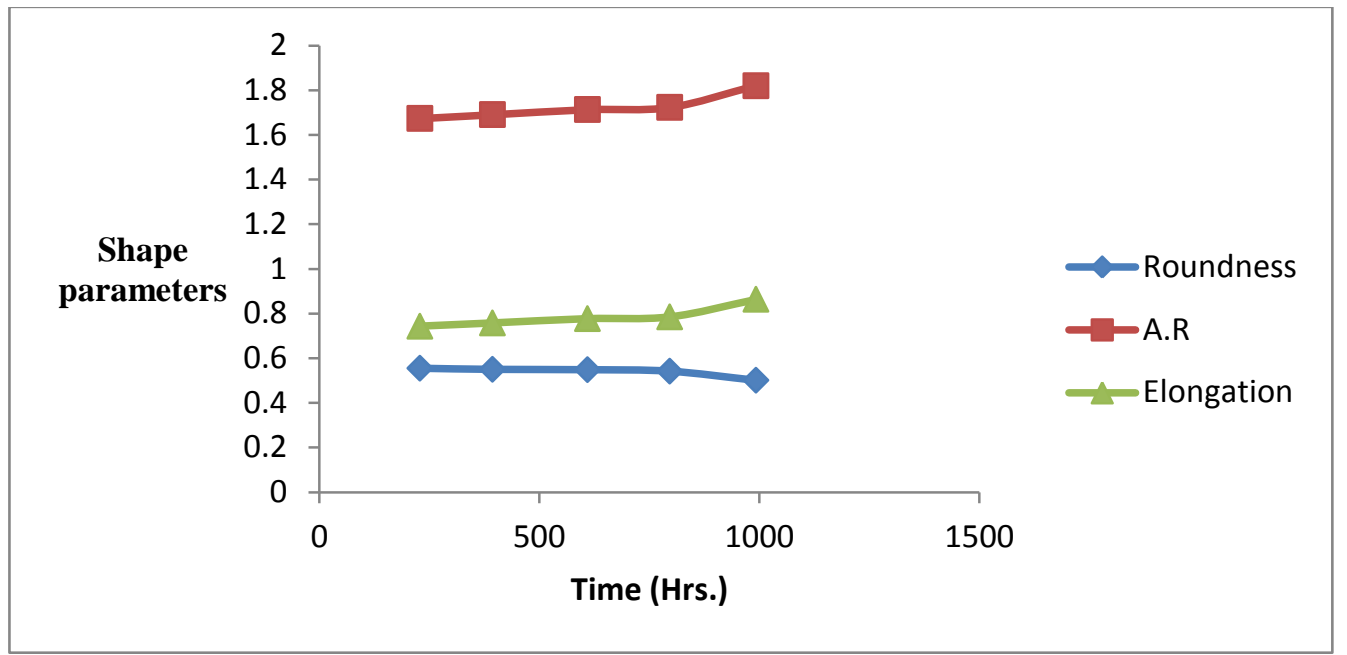

Figure 4:- Variation of shape parameters with time

Figure 4 depicts variation of shape parameters with time. It is evident from the figure that with passage of time the aspect ratio and the elongation of the wear particle increased while roundness of the wear particles decreased. Since the aspect ratio is increasing and the roundness is decreasing, we can infer that the abrading capacity of the wear particle increases.

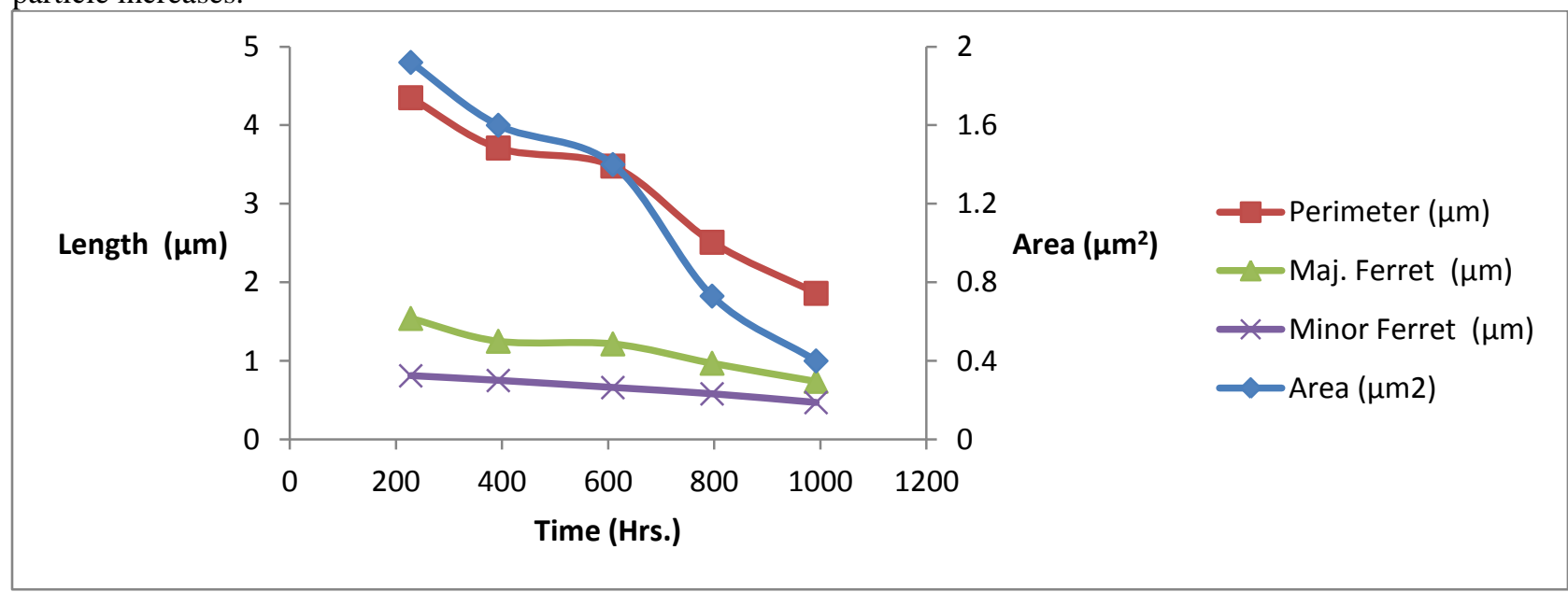

Figure 5:- Variation of size parameters with time

Figure 5 depicts variation of size parameters with time. It is evident from the figure that the all the size parameters viz. area, perimeter, major ferret and minor ferret decrease with passage of time.From the graphs, we can conclude that there is a gradual steady decrease with passage of time in case of major ferret and minor ferret. However, in case of area and perimeter of the wear particles there has been a sharp decrease after 600 hours of use of the transmission oil.

Table 2

\begin{tabular}{|l|l|l|l|l|l|}
\hline Type of particles & None & Few & Moderate & High & Debris morphology \\
\hline Rubbing wear particles & & & & $\checkmark$ & Very thin with smooth texture \\
\hline Surface fatigue particles & $\checkmark$ & & & & Thick particle with clearly defined periphery \\
\hline Rolling fatigue particles & $\checkmark$ & & & & Spherical particles \\
\hline Laminar particles & & & $\checkmark$ & & Thin particles with irregular contours \\
\hline Cutting wear particles & $\checkmark$ & & & & Curled with high aspect ratio \\
\hline Sliding wear particles & & & & $\checkmark$ & Sliding mark on surface with irregular periphery \\
\hline
\end{tabular}


Table 2 depicts presence of type of particle in the transmission oil and the classification of the wear particle based on the morphology of wear particle. It is evident from table 2 that the surface fatigue particles, rolling fatigue particles and the cutting wear particles were absent in the transmission oil. The laminar wear particles were moderate in quantity throughout the sampling process. The number of rubbing wear particles was high as compared to other wear particles at the beginning of the sampling process. However at the end of the sampling process, the number of sliding wear particles was high as compared to other wear particles.

\section{Conclusion:-}

This study demonstrates that ferrography is a powerful tool in condition monitoring as it can provide a great deal of information regarding wear particles which could indicate their individual histories.

The study has also shown that the shape parameters of the wear debris can be used to characterize the wear particle morphology. Of the shape parameters the prominent ones are roundness and the aspect ratio of the wear particles.With passage of time, there is an increase in number of wear particles which is attributable to deteriorating machinery condition and degradation of oil additives and their properties. In addition to it, there is a decrease in all the size parameters viz. area, perimeter, major ferretand minimum ferret which indicates that although the numbers of wear particles are increasing, their size keeps on decreasing. In the case of shape parameters, roundness decreases with time while there is an increase in aspect ratio and elongation. With increase in aspect ratio and decrease in roundness, we can infer that the abrading capability of the wear particle is increasing with time.

The wear debris generated reflects a composite wear mechanism of rubbing, cutting and severe sliding wear.In all the samples the wear mechanism has changed from normal rubbing wear to sliding wear with passage of time. There is no evidence of surface fatigue and rolling fatigue due to absence of chunky particlesand spherical particles respectively. Cutting wear particles are also absent indicating that the wear process is normal as compared to abnormal severe wear.

\section{Acknowledgement:-}

I am deeply indebted to Dr. Subrata KumarGhosh, Assistant professor, IIT (ISM) Dhanbad for his active guidance throughout the completion of the paper.

\section{References:-}

1. Z.Peng, T.B.Kirk (1998):Computer image analysis of wear particles in three-dimensions for machine condition monitoring; Wear 223,157-166

2. G. W. Stachowiak (1998):Numerical characterization of wear particle morphology and angularity of particles and surfaces;Tribology International Vol. 31, Nos 1-3, pp. 139-157

3. Kun Xu, A.R. Luxmoore, Farzin Deravi (1997): Comparison of shape features for the classification of wear particles; Engng. Applic. Artiff. Intel. Vol.10 No. 5 pp. 485-493

4. Jingqiu Wang, Xiaolei Wang(2013):A wear particle identification method by combining principal component analysis and grey relational analysis;Wear304,pp.96-102

5. ValdekMikli, HelmoKaerdi, PriitKulu, Michal Besterci (2001):Characterization of powder particle morphology; Proc. Estonian Acad. Sci. Eng., 7, 1, pp.22-34

6. Manoj Kumar, Parboti Shankar Mukherjee, Nirendra Mohan Mishra (2013):Advancement and current status of wear debris analysis for machine condition monitoring: A review, Industrial Lubrication and Tribology; Volume 65, Number 1, pp.3-11

7. ZhongxiaoPeng, Meiling Wang(2013):Three dimensional surface characterization of human cartilages at a micron and nanometer scale; Wear 301 pp.210-217

8. Dennis V. De Pellegrin, Gwidon W. Stachowiak(2002):Assessing the role of particle shape and scale in abrasion using sharpness analysis PART 1 Technique development;Wear 253, pp.1016-1025. 